\title{
EVALUACIÓN DE PROCEDIMIENTOS SIMPLIFICADOS EN LA CUANTIFICACIÓN VOLUMÉTRICA DE UNA PLANTACIÓN DE PINO
}

\author{
Evaluation of simplified procedures for quantifying wood volume \\ in a plantation of pine
}

Palabras clave: cubicación, inventario forestal, modelos de muestreo, ordenación forestal.

Key words: cubage, forest inventory, sampling models, forest management.
Eder Miguel Pereira ${ }^{1}$

José Imaña-Encinas ${ }^{2}$

Alba Valéria Rezende ${ }^{3}$

\section{RESUMEN}

La cuantificación del volumen sólido de madera en plantaciones forestales es imprescindible para la adecuada ordenación de los rodales y su correspondiente comercialización. Los métodos de muestreo utilizados tradicionalmente en los inventarios forestales son onerosos y dispendiosos siendo de fundamental importancia la obtención de métodos rápidos, baratos y precisos que faciliten la correspondiente cuantificación volumétrica sobre todo en plantaciones de áreas relativamente pequeñas. El objetivo de este trabajo consistió en comparar cuatro métodos de muestreo para determinar el volumen de madera de una plantación de Pinus. Tres de los métodos se fundamentaron exclusivamente en mediciones del diámetro y el cuarto correspondió al procedimiento de la cubicación de 553 árboles. En ocho parcelas circulares de $600 \mathrm{~m}^{2}$ cada una ubicadas aleatoriamente se obtuvo el volumen de madera por hectárea. Se observó que los métodos de obtención del volumen no presentaron diferencias significativas para un error máximo admisible de $10 \%$ considerado del nivel de significancia de $\alpha=0.05$. Los métodos permitieron la estimación confiable del volumen de madera por unidad de área, con una pequeña ventaja del método que utilizó el diámetro de Hohenadl.

\begin{abstract}
Quantification of wood volume in forests stands is necessary for forest management of plantations particularly with respect to the timber trade. Traditional sampling methods used in forest inventories are onerous and costly. Development of rapid, cheap and precise methods which facilitate the corresponding volumetric quantification are desirable. It was compared four sampling methods for determining wood volume in a Pinus plantation. Three of those methods are based exclusively on diameter measurement, whereas the fourth is based on wood volume. Eight randomly located $600 \mathrm{~m}^{2}$ plots were used where each method was applied to determine the wood volume per hectare. There were no significant differences in methods for determining wood volume with a permitted maximum error of $10 \%$, and a significance level of $\alpha=$ 0.05 . All methods provided reliable estimations of wood volume per unit area, although the Hohenadl diameter method performed slightly better.
\end{abstract}

\section{INTRODUCCIÓN}

Uno de los principales fundamentos del manejo y la ordenación de rodales forestales es la reali-

\footnotetext{
${ }^{1}$ Posgrado en Ciencias Forestales, Universidad de Brasilia. Brasilia, DF, Brasil. edermiguel@unb.br. Autor para correspondencia.

${ }^{2}$ Departamento de Ingeniería Forestal, Universidad de Brasilia. Brasilia, DF, Brasil. imana@unb.br

${ }^{3}$ Departamento de Ingeniería Forestal, Universidad de Brasilia, Brasilia, DF, Brasil. albavr@unb.br
} 
zación de inventarios forestales. El inventario forestal debe cuantificar y cualificar, correctamente, un rodal forestal compuesto por especies nativas o exóticas. Su actividad inherente sintetiza un trabajo que, por lo general, se realiza por algunos meses dependiendo del área que va a ser inventariada y envuelve costos elevados (ImañaEncinas, 1998). Estos factores hacen muchas veces impracticable la utilización del inventario forestal en empresas que poseen pequeñas áreas reforestadas. Sin embargo, al conocer el volumen de madera de una plantación forestal se valoriza eficiente y decididamente su producción maderera y sus productos en el contexto del uso social, ecológico y económico correcto, garantizando así la sostenibilidad de los recursos forestales.

De acuerdo con Imaña-Encinas (1998), el inventario forestal es el procedimiento de diagnóstico más eficiente de la producción de madera de un rodal sobre todo cuando su explotación está destinada a una constante y eficiente producción económica dentro del mercado maderero que, en el caso brasileño, considera ciclos de corta inferiores a ocho años para eucaliptos y entre veinte y venticinco años para pinos. Por lo tanto, es comprensible la necesidad de desarrollar y perfeccionar prácticas apropiadas de inventario forestal que permitan optimizar la obtención de datos de campo, disminuir los tiempos de ejecución y su interpretación dasométrica. Para Machado \& Figueiredo-Filho (2006), la mensura forestal en forma de censo, priorizando la cuantificación del volumen de todos los árboles en un rodal mismo ocupando una superficie relativamente pequeña, menor a 3000 ha, es muchas veces una operación impracticable, debido al tiempo necesario para su realización y, por ende, se convierte en una práctica económicamente inviable sobre todo cuando las áreas que van a ser inventariadas son mayores a 5000 ha.

Normalmente, los inventarios forestales que se realizan en plantaciones industriales se fundamentan en técnicas de muestreo estratificado que consideran como estratos prioritarios la especie plantada y su correspondiente edad (Pellico-Neto \& Brena, 1997; Soares et al., 2006). Por lo tanto, los fundamentos del muestreo deben permitir hacer evaluaciones tanto cuantitativas como cualitativas de áreas relativamente grandes, trabajando con eficiencia una serie de variables dasométricas solicitadas en la correspondiente planificación y el pertinente ordenamiento forestal.

En ese contexto, en las plantaciones industriales inventariadas por procedimientos de muestreo solamente una parte de la población arbórea es medida y los volúmenes calculados por unidad de área son extrapolados para el resto de la población. En esa extrapolación, los cálculos realizados con los principios de la estadística descriptiva ofrecen resultados muy consistentes y altamente confiables. Tomando en consideración la importancia de conocer con precisión el volumen de madera de una plantación, los procedimientos de muestreo son cada vez más importantes para que el sector forestal, originado de plantaciones industriales, sea cada vez más conocido y consecuentemente mejor administrado. Esa realidad pasa necesariamente por el análisis económico financiero de todas las actividades inherentes y de acciones orientadas al correspondiente manejo forestal, tanto en grandes empresas como en pequeñas propiedades forestales.

Los métodos tradicionales adoptados para estimar el volumen de madera en los inventarios forestales de plantaciones industriales involucran la utilización de ecuaciones volumétricas obtenidas mediante la cubicación de árboles apeados o de árboles que puedan ser procesados en pie (Prodan et al., 1997). Sin embargo, la práctica del inventario forestal tradicional necesita una enorme cantidad de tiempo y trabajo, además de ser una actividad compleja que incluye una serie de cálculos que no siempre son del total dominio de los técnicos ejecutores, lo que ocasiona un aumento en los correspondientes costos. Lo anterior genera que esa práctica sea difícil de realizar para propietarios que poseen pequeñas áreas arboladas con superficies menores a 3000 ha.

En el caso particular del gobierno brasileño, las políticas públicas incentivaron, sobre todo durante la última década, la actividad de la extensión 
forestal, lo cual permitió que pequeños y medios productores rurales puedan ingresar regularmente en la actividad productiva forestal principalmente en plantaciones de rápido crecimiento. Es muy posible que esta situación se repita en varias regiones de América Latina. En ese sentido, se hace necesario desarrollar procedimientos de muestreo para pequeñas áreas forestales, fundamentados básicamente en mediciones de diámetros, esto permite reducir el tiempo de ejecución cuando se compara con los sistemas de los inventarios tradicionales.

Con la filosofía de optimizar los tiempos de ejecución de los trabajos de campo y reducir los costos para el pequeño productor se identificó la posibilidad de ejecutar inventarios forestales midiendo solamente los diámetros de los árboles. El análisis interpretativo del volumen de madera se realizaría por medio de los diámetros medio cuadrático $\left(D_{g}\right)$, de Weise $\left(D_{w}\right)$ y el de Hohenadl $\left(D_{H}\right)$ (Imaña-Encinas, 2011) que permitan presentar la confiabilidad de los resultados pertinentes. En ese contexto para inventariar un pequeño bosque plantado se compararon tres modelos de muestreo utilizando los parámetros dasométricos: diámetro medio cuadrático $\left(\mathrm{D}_{\mathrm{g}}\right)$, diámetro de Weise $\left(\mathrm{D}_{\mathrm{W}}\right)$, diámetro de Hohenadl $\left(\mathrm{D}_{\mathrm{H}}\right)$ conjuntamente con la realización de una cubicación del área experimental. La finalidad del estudio fue comparar el volumen de madera por hectárea obtenido por los tres métodos con el cálculo del volumen real conseguido por medio de la cubicación.

\section{MATERIALES Y MÉTODOS ÁREA DE ESTUDIO}

El área de estudio fue una plantación de Pinus caribaea var. hondurensis (Sénécl.) W.H.G. Barrett \& Golfari, de 15.3 ha, localizada en el municipio de Caravelas en la región sur del Estado de BahiaBrasil, situándose geográficamente en las coordenadas de latitud Sur $17^{\circ} 49^{\prime} 41^{\prime \prime}$ y de longitud Oeste 39²6'39" (Figura 1). De acuerdo con Embrapa (2006) en la región predominan suelos del tipo Latos suelo Amarillo con horizonte A moderado, textura media y relieve plano. El clima según la

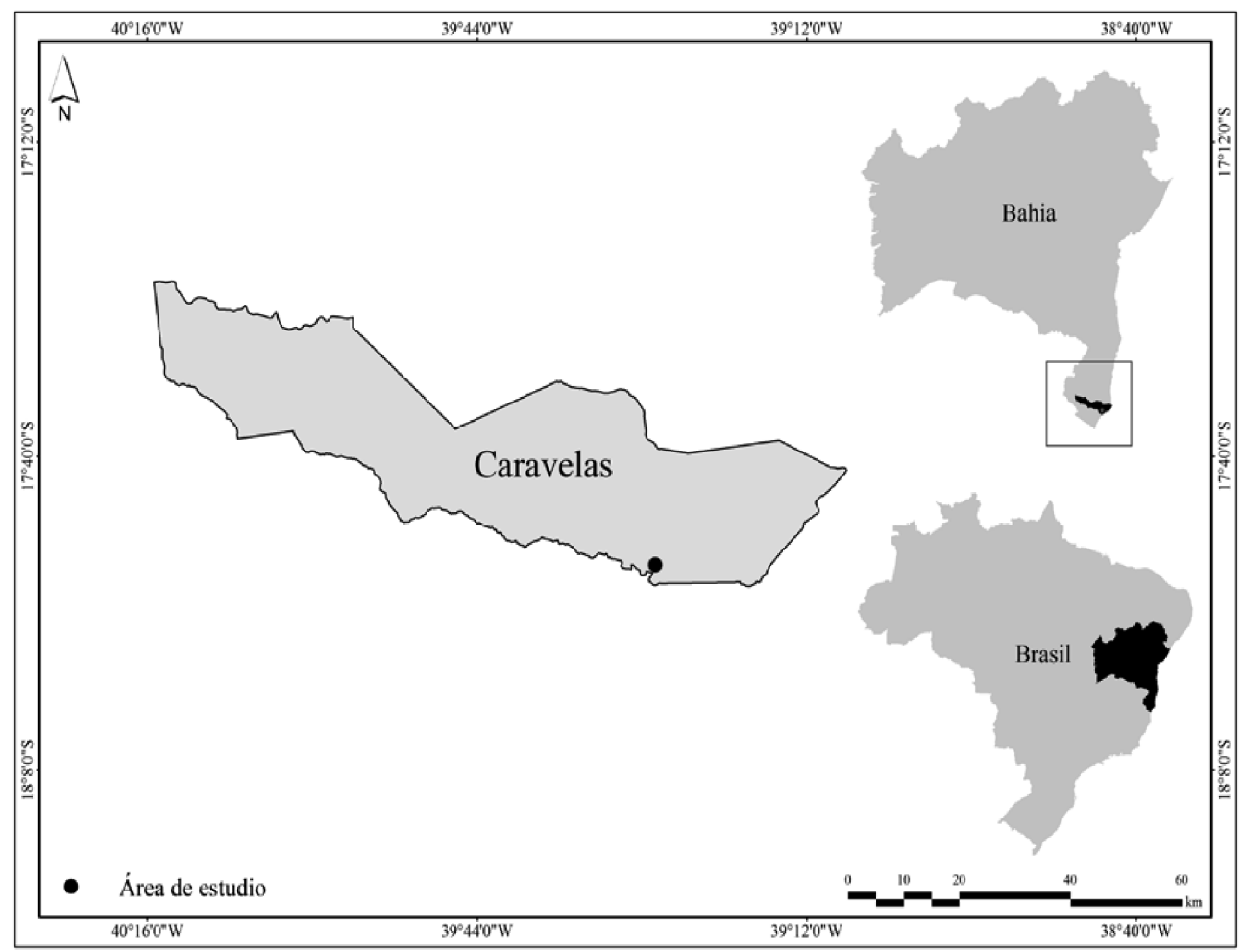

Figura 1. Localización del área de estudio. 
clasificación de Köppen es del tipo Aw que corresponde al clima tropical húmedo con estación lluviosa en el verano y seca en el invierno.

\section{MUESTREO ESTADÍSTICO}

Se establecieron aleatoriamente ocho parcelas circulares de $600 \mathrm{~m}^{2}$ cada una. El radio de la parcela fue de $13.82 \mathrm{~m}$ donde se deberían encontrar aproximadamente cien árboles por parcela en un espaciamiento de $3 \times 2 \mathrm{~m}$. Se siguió el procedimiento de la selección del muestreo aleatorio simple, totalizando un área experimental de $4800 \mathrm{~m}^{2}$ de una superficie total de $15.3 \mathrm{ha}$. Al momento del estudio la plantación tenía la edad de once años y medio. El DAP fue medido en el total de la muestra observada, correspondiendo a 553 árboles en pie. Posteriormente todos los árboles se cortaron a la altura de $0.20 \mathrm{~m}$ del suelo para proceder a cubicarlos.

\section{MEDICIÓN DE VARIABLES}

En cada una de las ocho parcelas, se marcaron y numeraron todos los árboles en pie, a los que también se les midió el diámetro a $1.30 \mathrm{~m}$ de altura del suelo (DAP) con una forcípula. Las alturas totales de los árboles apeados se determinaron con una cinta métrica de $20 \mathrm{~m}$ después de ser cortados. Durante el procedimiento de la cubicación se midieron con forcípula los diámetros a las alturas de 0.10 , $0.30,0.50,0.70,0.90,1.10,1.30,2.00,3.00 \mathrm{~m}$ y así sucesivamente hasta el tope de la altura total.

Con el objetivo de obtener el volumen total real de los árboles individualmente en la cubicación se utilizó la fórmula de Smalian en el cálculo de las correspondientes secciones del tronco. Los volúmenes individuales de cada sección del tronco se sumaron para la obtención del volumen real del árbol. Al total de los volúmenes de los árboles apeados se les denominó volumen real de la parcela. El valor medio de las ocho parcelas se multiplicó por el factor 16.67 para extrapolar su resultado a una hectárea (Imaña-Encinas et al., 2002) identificando ese parámetro como el volumen real por hectárea.

\section{PROCEDIMIENTOS EVALUADOS}

Con los diámetros medidos en los 553 árboles en pie se calculó el diámetro medio cuadrático $(\mathrm{Dg}$; Ec. 1), el diámetro de Weise $\left(\mathrm{D}_{\mathrm{w}}\right)$ y el diámetro de Hohenadl $\left(\mathrm{D}_{\mathrm{H}}\right)$. La cubicación se la realizó independientemente en las ocho parcelas correspondientes. Los diámetros dasométricos se definieron como:

\section{Diámetro medio cuadrático (Dg)}

Su fórmula de cálculo fue:

$$
D g=\sqrt{\frac{\sum_{i=1}^{N}(D A P)^{2}}{n}}
$$

considerando en su determinación todos los árboles de la parcela correspondiente. Posteriormente fue realizada la selección de cinco árboles por parcela de tuvieran sus diámetros más próximos al $\mathrm{D}_{\mathrm{g}}$ calculado. De esos cinco árboles que ya habían pasado por el proceso de la cubicación se extrajo, del banco de datos, el valor del volumen de madera correspondiente. El valor medio de esos cinco árboles se extrapoló para la parcela y la hectárea respectivamente.

\section{Diámetro de Weise $\left(\mathrm{D}_{\mathrm{w}}\right)$}

En cada parcela, teniendo los diámetros organizados en orden creciente, se seleccionó el árbol que se localizó en la posición del $60 \%$ a fin de identificar el $\mathrm{D}_{\mathrm{W}}$ (Prodan, 1965; Imaña-Encinas, 2004, 2011) Consecuentemente, el árbol de esa posición ya tenía el valor correspondiente del volumen obtenido por la cubicación. Se obtuvo posteriormente el valor medio de los cinco árboles más próximos al Dw encontrado en igual procedimiento al descrito con el Dg. El volumen encontrado se extrapoló para la parcela, multiplicando su valor por 67 (número medio de árboles encontrados por parcela). El resultado se multiplicó por el factor 16.67 para obtener el volumen de madera por hectárea (Imaña-Encinas et al., 2002). 


\section{Diámetro de Hohenadl ( $\left.\mathrm{D}_{\mathrm{H}}\right)$}

De la misma lista de diámetros por parcela organizados en orden creciente, se escogieron los árboles de las posiciones de los 16 y $84 \%$, respectivamente determinándose los diámetros externos de Hohenadl (D y $\mathrm{D}_{+}$respectivamente). Se calculó para esos dos diámetros el correspondiente diámetro medio equivalente al $\mathrm{D}_{\mathrm{H}}$ (Prodan et al., 1997; Scolforo \& Mello, 1997; Imaña-Encinas, 2003). El procedimiento para encontrar el volumen por hectárea siguió lo establecido para el $\mathrm{D}_{\mathrm{w}}$.

Para la evaluación de los métodos de estimación del volumen por unidad de área se optó por utilizar comparaciones de medias. El error en porcentaje (E\%; Ec. 2) de los diferentes procedimientos comparados con la cubicación fue obtenido por la expresión:

$$
E(\%)=\frac{\left(V o l_{\mathrm{Re} a l}-V o l_{\text {Tratamiento }}\right)}{V o l_{\mathrm{Re} a l}} \cdot 100 \quad \text { Ec. } 2
$$

\section{RESULTADOS}

Con las mediciones obtenidas de los DAP se construyó el histograma de frecuencia de los diámetros (Figura 2) con amplitud de clase de $2.5 \mathrm{~cm}$ que permitió mostrar su distribución diamétrica correspondiente a la hectárea. Se verificó que los valores de los diámetros medidos del total de la muestra observada siguen la curva de tendencia de una distribución normal presentando pocos individuos en el extremo superior, con una asimetría negativa (valor de la moda mayor que la media aritmética). La mayor concentración de individuos se presentó en las cuatro primeras clases diamétricas $(73 \%$ del total de los individuos por hectárea). En la clase diamétrica de 12.5 a $15.0 \mathrm{~cm}$ se ubicaron el DAP medio aritmético, la moda, el Dg, el DAP medio ponderado y el $\mathrm{D}_{\mathrm{H}}$ en una variación de menos de $2 \mathrm{~cm}$ entre los valores extremos calculados. El Dw quedó localizado en la clase diamétrica de 15 a $17.5 \mathrm{~cm}$.

Entre las características estadísticas de las ocho parcelas se observó que el DAP medio aritmético varió en una amplitud de $4.53 \mathrm{~cm}$ de la desviación estándar presentando una media general de $13.74 \mathrm{~cm}$ de los 553 DAP con un rango entre 5.4 y $25.5 \mathrm{~cm}$. El coeficiente de variación identificó valores entre 31.20 y $38.04 \%$ en las diversas parcelas, lo cual demuestra que las parcelas de 600 $\mathrm{m}^{2}$ tuvieron comportamiento semejante y de esa forma se puede inferir que las parcelas fueron plenamente representativas de la plantación forestal. El coeficiente de variación del DAP tuvo una variación entre las parcelas de apenas $6 \%$, mostrando bastante homogeneidad en la plantación. En

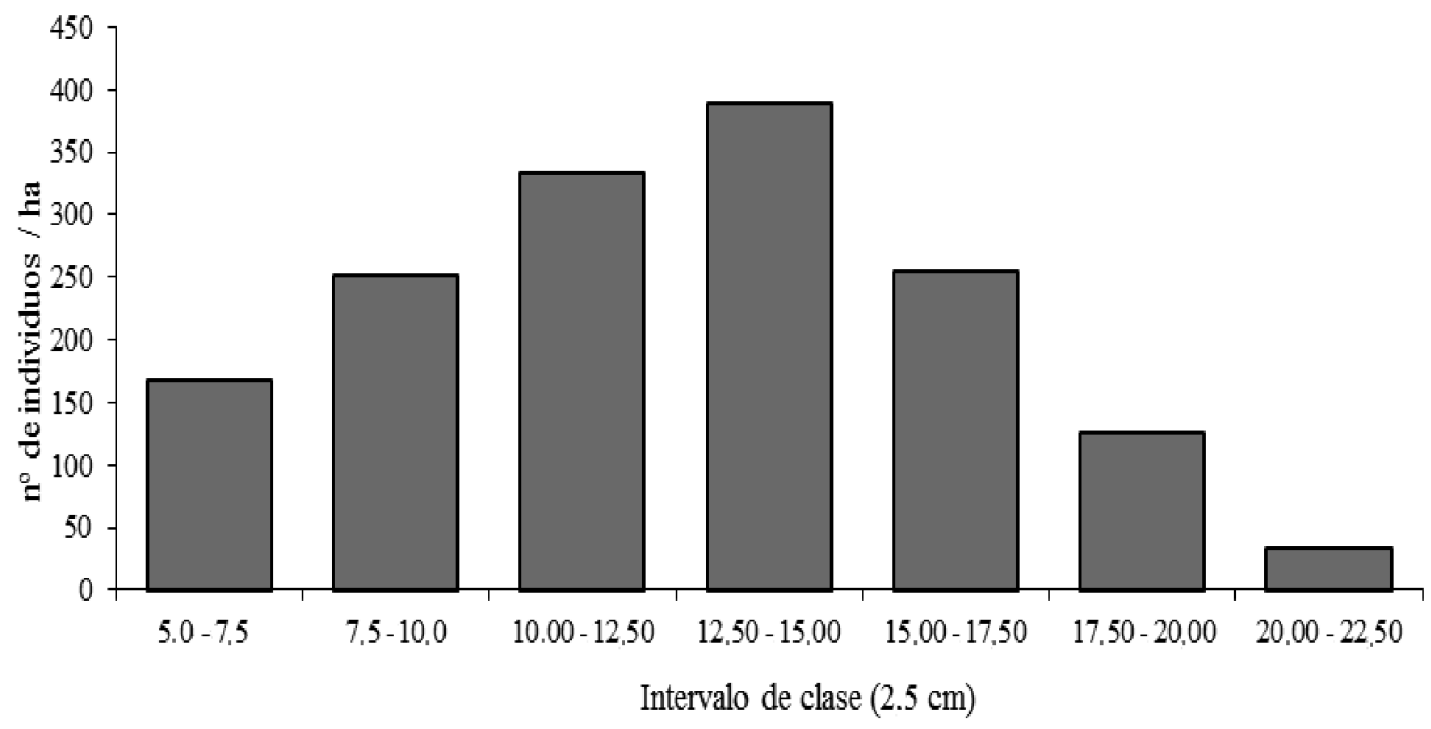

Figura 2. Distribución diamétrica de los árboles extrapolada para la hectárea 
ese sentido el DAP medio de la muestra medida $(13.74 \mathrm{~cm})$ corresponderá al árbol medio de la plantación, presumiéndose que el rodal aún está en plena fase de crecimiento.

Un aspecto perceptible fue la poca variación mostrada entre los atributos medidos en las respectivas parcelas. Este comportamiento puede indicar la existencia de una homogeneidad de crecimiento de la plantación, característica que facilitó eficientemente la obtención de los estimadores de la variable de interés.

Por la cubicación de los troncos de los 553 árboles apeados, la fórmula de Smalian se mostró eficiente presentando un tiempo relativamente corto en la obtención de los datos de campo. El coeficiente de variación del total de los árboles apeados presentó un valor de $20.90 \%$ y una media aritmética de $0.3831 \mathrm{~m}^{3} / a ́ r b o l$.

El resultado del cálculo de los volúmenes obtenidos en $\mathrm{m}^{3}$ por parcela, sus valores medios, coeficiente de variación y error en relación con el volumen obtenido por medio de la cubicación (considerado como testigo) se muestra en la tabla 2. Se observa que los cuatro métodos analizados mostraron ser consistentes obteniéndose resultados con una pequeña variación entre sí. Esos resultados posibilitaron la comparación de los volúmenes obtenidos por parcela, lo que permite confirmar por el coeficiente de variación del volumen, que oscilando entre los valores de 19.70 a $22.68 \%$, se trata de una población muy próxima a ser homogénea. En ese sentido, las parcelas medidas pueden ser consideradas plenamente representativas del total de la plantación y sus resultados inferidos satisfactoriamente a nivel de hectárea en relación al volumen.

El método que más se aproximó al volumen real (testigo) al analizar los valores correspondientes y los errores generados fue el del diámetro de Hohenadl $\left(\mathrm{D}_{\mathrm{H}}\right)$ que proporcionó errores de menos de $1 \%$ (Tabla 2) y en ese sentido el $\mathrm{D}_{\mathrm{H}}$ muestra la mayor aproximación al diámetro medio aritmético del tratamiento testigo. El método del diámetro medio cuadrático presentó un error de $-3.6 \% \mathrm{y}$ el procedimiento del diámetro de Weise mostró la mayor diferencia en relación al testigo que fue de

Tabla 2. Volúmenes estimados en $\mathrm{m}^{3}$ mediante diferentes procedimientos de cuantificación volumétrica.

\begin{tabular}{ccccc}
\hline Parcela & $\begin{array}{c}\text { Diámetro } \\
\text { cuadrático }\end{array}$ & $\begin{array}{c}\text { Diámetro } \\
\text { Weise }\end{array}$ & $\begin{array}{c}\text { Diámetro } \\
\text { Hohenadl }\end{array}$ & $\begin{array}{c}\text { (Testigo) } \\
\text { Cubicación }\end{array}$ \\
\hline 01 & 13.1584 & 13.7421 & 12.7430 & 12.5249 \\
02 & 19.3665 & 19.0078 & 17.0188 & 16.8550 \\
03 & 12.0567 & 13.2966 & 11.8891 & 11.7105 \\
04 & 21.7645 & 23.6280 & 22.2234 & 22.6108 \\
05 & 13.8652 & 15.2911 & 13.6525 & 13.4671 \\
06 & 14.2269 & 15.6900 & 14.0192 & 13.8184 \\
07 & 15.6737 & 17.2856 & 15.4550 & 15.2236 \\
08 & 15.0709 & 16.8203 & 14.8514 & 14.6381 \\
\hline media/parcela & 15.6504 & 16.8203 & 15.2326 & 15.1061 \\
media/hectárea & 260.8398 & 280.3379 & 253.8775 & 251.7677 \\
CV \% & 21.05 & 19.70 & 21.57 & 22.68 \\
error \% & -3.60 & -11.35 & -0.83 & \\
\hline
\end{tabular}


$-11.35 \%$. De acuerdo con Imaña-Encinas (2004) el $\mathrm{D}_{\mathrm{w}}$ corresponde al árbol que debe llevar con mucha aproximación el volumen medio representativo por hectárea de plantaciones homogéneas.

Un aspecto importante para ser mencionado sobre los procedimientos de estimación del volumen por los métodos de los diámetros medio cuadrático, de Weise y de Hohenadl, fue que el número de árboles que fueron apeados y cubicados en este estudio se restringió a cuarenta individuos por procedimiento (cinco por parcela respectivamente). Si se considera que el error calculado fue relativamente pequeño entre los procedimientos utilizados en relación con la cubicación, se mostró que estos procedimientos pueden ser plenamente representativos y aplicables en plantaciones de superficies relativamente pequeñas.

\section{DISCUSIÓN}

El DAP medio aritmético del presente trabajo fue de $13.73 \mathrm{~cm}$ valor que demuestra coincidir con los obtenidos por Schneider et al. (2008), que encontró $13.95 \mathrm{~cm}$ a la edad de once años en plantaciones de Pinus taeda en la región de Telêmaco Borba estado de Paraná (Brasil). En plantaciones de Pinus en el sur del Brasil fueron registrados a la edad de once años $14 \mathrm{~cm}$ de DAP y en la edad de treinta años $35 \mathrm{~cm}$ (Figueiredo-Filho et al., 2006). Consecuentemente los resultados del DAP medio obtenidos se encuadran plenamente a las informaciones bibliográficas correspondientes.

Figueiredo-Filho et al. (2006) trabajando en plantaciones de Pinus en la flona (bosque nacional) de Irati en el estado de Paraná, con edades entre ocho y cuarenta años constató una variación media del diámetro para la edad de once años alrededor de $39 \%$, por lo tanto, el volumen presenta una variación media del 25\%. Estos resultados se aproximan bastante a los encontrados en el presente trabajo (Tabla 2) y son semejantes a los encontrados por Sanquetta et al. (2003), en los que se evaluó una plantación de $P$. taeda a los doce años en la región norte del estado de Paraná. Por su parte, Inoue et al. (2011) estudiaron el efecto del espaciamiento sobre el crecimiento de la variable diámetro y obtuvo un coeficiente de variación del 29\% para plantaciones de Pinus de diez años de edad, valor que se ubica próximo al encontrado en el presente estudio.

En relación con la eficiencia de uso de la fórmula de Smalian en trabajos de trozado y cubicación de troncos de Eucalyptus en plantaciones industriales Machado \& Figueiredo-Filho (2006) y Andrade et al. (2009) encontraron mayor eficiencia en la obtención de datos de campo cuando se los compararon con el uso de las fórmulas de Huber y Newton.

El volumen medio obtenido por hectárea que se encontró en el presente trabajo fue de aproximadamente $250 \mathrm{~m}^{3}$ Figueiredo-Filho et al. (2006) encontraron un valor medio de $240 \mathrm{~m}^{3}$ y Thomas et al. (2006) un valor de $258 \mathrm{~m}^{3} / \mathrm{ha}$ en plantaciones de once años. Inoue et al. (2011) encontraron $285 \mathrm{~m}^{3} / \mathrm{ha}$.

El procedimiento de cálculo para estimar el volumen de madera en pie por medio de datos exclusivos de DAP se mostró plenamente eficiente. En ese sentido será posible identificar uno de los diámetros medios dasométricos de interés y en apenas cinco árboles que llevasen valores próximos al DAP encontrado, podrán ser cubados. Esto permitirá una enorme economía de tiempo y recursos además de poder proceder con un cálculo sumamente sencillo en su correspondiente identificación.

\section{CONCLUSIONES}

Los métodos no presentaron diferencias significativas en el cálculo del volumen de madera de la parcela. Por lo tanto, pueden ser consistentemente utilizados para las estimaciones del volumen por unidad de área en plantaciones de pequeñas a medianas superficies, desde que no sobrepasen tres mil hectáreas de plantación homogénea.

De los métodos estructurados en las mediciones de diámetro, la estimación del volumen se restringe a la cubicación de hasta cinco árboles que lleven aproximadamente el diámetro dasométrico $\mathrm{D}_{\mathrm{W}}$ o $\mathrm{D}_{\mathrm{H}}$ correspondiente. 


\section{REFERENCIAS BIBLIOGRÁFICAS}

Andrade, V.C.L., Calegario, N., Souza, A.L., Rezende, J.L.P., Truguilho, P.F., Rosado, S.C.S., \& Silva, J.R.M. (2009). Uso de sólidos geométricos para gerar equações de forma do tronco de árvores em pé. Scientia Forestalis, 37, 299-311.

Empresa Brasileira de Pesquisa Agropecuária [Embrapa]. (2006). Sistema brasileiro de classificação de solos. Rio de Janeiro: Embrapa Solos. 306 p.

Figueiredo-Filho, A., Dias, A.N., \& Watzlawick, L.F. (2006). Inventário das florestas plantadas na Floresta Nacional de Irati. Irati: Unicentro. $103 \mathrm{p}$.

Imaña-Encinas, J. (1998). Dasometría práctica. Brasilia: Editora Universidade de Brasilia. 117 p.

Imaña-Encinas, J. (2003). Determinação dos diâmetros de Hohenadl. En Programa de Pós-Graduação em Ciências Florestais (ed.), Anais, $9^{\circ}$ Congresso Florestal Estadual do Rio Grande do Sul (pp. 1-9). Nova Prata: Universidade Federal de Santa Maria, Departamento de Engenharia Florestal.

Imaña-Encinas, J. (2004). Identificación simplificada de diámetros dasométricos. En P.R. Schneider \& C.A.G. Finger (eds.), $3^{\circ}$ Simpósio Latino-Americano sobre manejo florestal, Santa Maria, Anais (pp. 222-225). Santa Maria: Universidade Federal de Santa Maria.

Imaña-Encinas, J. (2011). Slides de aula da disciplina dendrometria. Brasília: Universidade de Brasilia, Departamento de Engenharia Florestal. $231 \mathrm{p}$.

Imaña-Encinas, J., da Silva, G.F., \& Kishi, I.T. (2002). Variáveis dendrométricas. Comunicações Técnicas Florestais (Vol. 4, No. 1). Brasília: Universidade de Brasília, Departamento de Engenharia Florestal. 102 p.
Inoue, M.T., Figueiredo-Filho, A., Araújo, A.J., \& Lima, R. (2011). Crescimento juvenil de $P i$ nus taeda em função do espaço vital de crescimento. Revista Floresta, 41, 57-62.

Machado, S.do A., \& Figueiredo-Filho, A. (2006). Dendrometria ( $2^{\text {da }}$ ed.). Guarapuava: Unicentro. $316 \mathrm{p}$.

Péllico-Neto, S., \& Brena, D.A. (1997). Inventário Florestal. Curitiba: Universidade Federal do Paraná. 245 p.

Prodan, M. (1965). Holzmesslehre. Frankfurt: J.D. Sauerlaender's Verlag. 644 p.

Prodan, M., Peters, R., Cox, F., \& Real, P. (1997). Mensura Forestal. San José de Costa Rica: GTZ-IICA. 586 p.

Sanquetta, C.R., Arce, J.E., Mello, A.A., Silva, E.Q., Barth-Filho, N., \& Matoski, S.L.S. (2003). Produção de madeira livre de nós em povoamentos de Pinus taeda em função da densidade de plantio. Cerne, 9, 129-140.

Schneider, P.R., Finger, C.A.G., Bernett, L.G., Schneider, P.S.P., \& Fleig, F.D. (2008). Estimativa dos parâmetros da função de densidade probabilística de Weibull por regressão aninhada em povoamentos desbastados de Pinus tae$d a$ L. Ciência Florestal, 18, 381-392.

Scolforo, J.R.S., \& Mello, J. M. (1997). Inventário florestal. Lavras: Universidade Federal de Lavras. 310 p.

Soares, C.P.B., Paula-Neto, F., \& Souza, A.L. (2006). Dendrometria e inventário florestal. Viçosa: Universidade Federal de Viçosa. 276 p.

Thomas, C., Andrade, C.M., Schneider, P.R., \& Finger, C.A.G. (2006). Comparação de equações volumétricas ajustadas com dados de cubagem e análise de tronco. Ciência Florestal, 16, 319-327. 